\title{
Towards a general theory of fighting arts
}

\author{
Wojciech J. CYNARSKI ${ }^{*}$ \\ Faculty of Physical Education, University of Rzeszow, Rzeszow (Poland)
}

\section{Introduction}

The fighting arts is an interesting area for researchers from many scientific disciplines around the world. Scientific investigations need to choose appropriate theory, language and methods. The first theory that gives us a special language is the Humanistic Theory of Martial Arts, HTMA. The second is the Anthropology of Martial Arts, AMA (Cynarski, 2012).

\section{Aim}

The problem considered in this study is how we should study the martial arts \& combat sports \& systems, or 'combatives'; what theoretical framework should we use. It is the author's proposal for a scientific perspective which should be useful; an elaboration the Humanistic Theory of Martial Arts (HTMA) and definitions, the General Theory of Fighting Arts (GTFA), and the Anthropology of Martial Arts.

\section{Methodology}

Participant observation and qualitative content analysis of the literature were used. Methodology of research (in the area of the fighting arts) was also analyzed.

\section{Results}

\section{A. The Humanistic Theory of Martial Arts - HTMA}

This new-paradigmatic theory gives us the Language of the Theory. Terms, such as physical culture, martial arts, ways of martial arts (Budo), combat sports and combat systems, are important to the creation of the General Theory of Fighting Arts (GTFA).

\section{B. The Anthropology of Martial Arts - AMA}

AMA is the second step in the development of a scientific framework for Combat Sports \& Martial Arts. From the anthropological perspective of the human being, their personal, physical and psychological being is the centre of attention. The body is not undervalued here, but bodily practice is related to the path of spiritual development.

The pursuit of perfection affects both the skills of hand-to-hand fighting and of wielding weapons, as well as morality and the pursuit of full humanity. Grand Masters of martial arts schools introduce philosophical elements from various philosophical and religious traditions in their training.

Wally Strauss' philosophy of Ido ('perpetual movement', 'endless road', or 'infinite way') is particularly interesting (Cynarski, 2009), and adopted by a group of today's warriors of the 
pathway. This philosophy unites new Budo with the European chivalric ethos, the ethics of brotherhood and personal self-realization.

C. General Theory of Fighting Arts - GTFA

In the GRFA we can study and explain a broad area of fighting arts and related problems. These are:

1. Cultural areas of martial arts \& combat sports \& combat systems (according to AMA and HTMA);

2. Teaching concepts (technical and tactical issues), combat strategy (in self-defence and in combat sports), aspects of real fighting - interdisciplinary;

3. Bio-technical issues - from perspective of sport sciences.

The Research methodology for traditional East Asian martial arts requires its own practice and a high level of sophistication - at best, in more than one martial art. It is a qualitative method of participant observation associated with the experience of metaphysical dimensions. This special method is the 'shugyo' (Jap. shügyō) - psychophysical study and experience from practicing psychophysical systems. Michael Maliszewski was one of first researchers using this method ( $c f$. Maliszewski, 1996; Cynarski, 2012a; Spencer, 2012).

\section{Conclusion}

The GTFA is the sum of HTMA (humanistic, social and cultural issues), AMA (philosophical, cultural and biological approaches), and a part of Sport Sciences, for coaching, bio-technical and bio-medical issues, the methodology of teaching, etc. The GTFA now created should give us the opportunity to research and explain complicated problems of fighting arts, according to this new, holistic paradigm.

\section{References}

Cynarski, W.J. (2009). Martial Arts - Ido \& Idokan. Rzeszow:IPA [in Polish].

Cynarski, W.J. (2012). Anthropology of Martial Arts. Studies and Essays from Sociology and Philosophy of Martial Arts. Rzeszow: Rzeszow University Press [in Polish].

Maliszewski, M. (1996). Spiritual Dimensions of the Martial Arts. Rutland-Tokyo: C.E. Tuttle Company.

Spencer, D.C. (2012). Ultimate fighting and embodiment. Violence, gender, and Mixed Martial Arts. New York: Routledge.

Key words: Fighting arts; martial arts; combat sports; physical culture; anthropology. 